\title{
Visualization of Primary Particles in a Tablet Based on Raman Crystal Orientation Mapping
}

Kei Moriyama ${ }^{1 *}$, Hiroki Onishi ${ }^{1}$ and Hiromi Ota ${ }^{2}$

${ }^{1}$ Shujitsu University, School of Pharmacy, 1-6-1 Nishigawara, Naka-ku, Okayama 703-8516, Japan

${ }^{2}$ Advanced Science Research Center, Okayama University, 1-1-1 Tsushima-naka, Kita-ku, Okayama 700-8530, Japan

\begin{abstract}
Objective: The morphology of the primary particles in the active pharmaceutical ingredient (API) is one of the most important determinants for formulation function. However, it has not been possible to visualize primary particles in a tablet using various imaging methods, including spectroscopic mappings, because these particles usually exist as aggregated clusters in tablets. We revealed that the Raman spectrum of crystalline particles is determined depending upon the angle between the crystallographic axis and the polarization direction of the excitation laser. In this paper, we report a method to visualize primary particles within the aggregated cluster based on the Raman spectral change on the boundary of primary particles.
\end{abstract}

Method: Metformin hydrochloride was chosen as a model API for this study. The crystal structure of metformin hydrochloride was solved using X-ray crystal structure analysis. The Raman spectra of metformin hydrochloride crystal along the $x y z$ axes were recorded and resolved into components along the $a b c$ axes. Using the abc components, Raman mapping of metformin hydrochloride in tablets was performed to visualize the crystal orientation at each data point.

Results: Metformin hydrochloride crystals recrystallized from water/ethanol formed a primitive monoclinic cell. Datasets of five distinct peak areas from the metformin hydrochloride Raman spectrum were used for analyses. Raman crystal orientation mapping (RCOM) from the tablet cross-section provided an image of primary particles within the aggregation cluster of metformin hydrochloride in the tablet.

Conclusion: Based on the RCOM, we developed a visualization method for primary API particles in tablets. Because the morphology of primary particles is the key factor of formulation function, this method would contribute to better formulation development and quality control.

Keywords: Raman spectroscopy; Raman mapping; Primary particle; Formulation; Metformin

\section{Introduction}

Raman chemical imaging has become a powerful tool for understanding solid formulations by visualizing the dispersion of active pharmaceutical ingredients (API) [1-6] or excipients [7]. However, particularly in high dose formulations, the image of API is usually the image of the aggregation cluster. At this stage, it is not possible to resolve the image to the level of the primary particles by Raman chemical imaging. The morphology of primary particles is one of the key factors in determining the formulation functions $[8,9]$. Development of imaging techniques for the primary particles in the aggregation would greatly contribute to formulation research and quality control.

Using an antiepileptic agent, phenytoin, as a model API, we have revealed that the peak area of the Raman spectrum changes depending upon the angle of the crystallographic axes and polarization direction of the excitation laser [10]. In our report, we showed that the crystal face of the API can be identified according to the combination of Raman spectra where the polarization direction was horizontal and vertical to the morphological long axis of a target face. Applying this to Raman mapping, we suggested that mapping of the angle between the crystallographic axes and the polarization direction could be achieved. Because the axes orientation within a certain primary particle is constant against the polarization direction and this obviously changes on the boundary between particles, the Raman mapping of the axes orientation (Raman crystal orientation mapping (RCOM)) could be used to visualize the primary particle image within the aggregated cluster of API.

In this report, we showed the first attempt at RCOM and the primary particle imaging for a high dose solid formulation of antidiabetic agent, metformin hydrochloride. We discussed the feasibility of this imaging method for better formulation development and quality control.

\section{Materials and Methods}

\section{$\mathrm{X}$-ray single-crystal structural analysis of metformin hydrochloride}

Metformin hydrochloride (Tokyo Kasei, Tokyo, Japan) (Figure 1A) was recrystallized from water/ethanol to obtain a crystal for $\mathrm{X}$-ray single-crystal structural analysis. A colorless prism crystal of metformin hydrochloride (Figure $1 \mathrm{~B}$ ) having approximate dimensions of $0.120 \times 0.100 \times 0.050 \mathrm{~mm}$ was mounted in a loop. All measurements were made on a Rigaku Saturn724 diffractometer (Rigaku Corporation,

*Corresponding author: Kei Moriyama, Shujitsu University, School of Pharmacy, 1-6-1 Nishigawara, Naka-ku, Okayama 703-8516, Japan, Tel: +81 862718155 Fax: +81 862718155 ; E-mail: moriyama@shujitsu.ac.jp

Received November 03, 2015; Accepted December 28, 2015; Published December 30, 2015

Citation: Moriyama K, Onishi H, Ota H (2015) Visualization of Primary Particles in a Tablet Based on Raman Crystal Orientation Mapping. Pharm Anal Acta 6: 453 doi:10.4172/21532435.1000453

Copyright: @ 2015 Moriyama $\mathrm{K}$ et al. This is an open-access article distributed under the terms of the Creative Commons Attribution License, which permits unrestricted use, distribution, and reproduction in any medium, provided the original author and source are credited. 

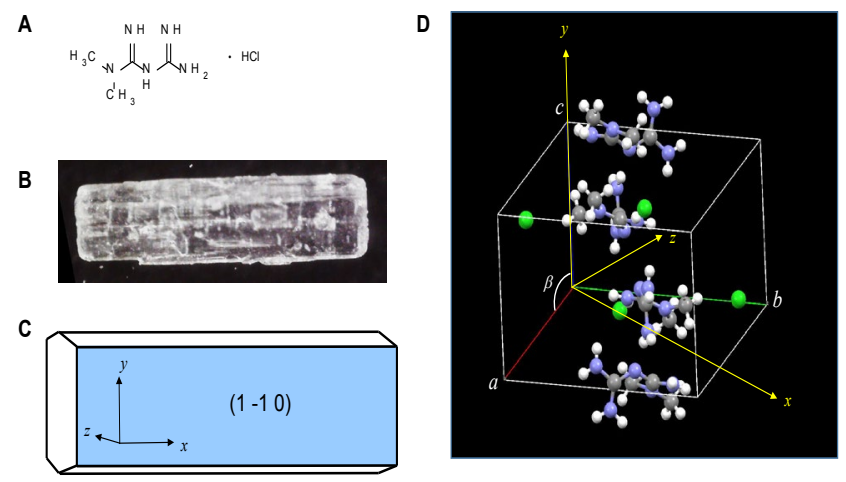

Figure 1: (A) Chemical structure of metformin hydrochloride, (B) microscopic view of metformin hydrochloride crystal, (C) definition of the $x y z$ axes of coordinates on the morphology of metformin hydrochloride crystal, (D) the unit cell and the abc axes of metformin hydrochloride crystal. Yellow axes indicate $x y z$ axes in $(C)$.

Tokyo, Japan) using a multi-layer mirror monochromated Mo-Ka radiation.

\section{Raman spectra measurement and resolution into abc components}

The $x y z$ axes of coordinates were defined so that the $x y$ plane was on the $(1-10)$ face and the $x$ axis was parallel to the morphologically long axis of the crystal (Figure 1C). Raman spectra were recorded on an inVia Raman microscope system (Renishaw plc, Gloucestershire, UK) equipped with a Leica microscope and a $785 \mathrm{~nm}, 300 \mathrm{~mW}$ excitation lasers. 50 $\underline{\mathrm{X}}$ objective lens was used and the laser exposure time was $1 \mathrm{~s}$. The datasets of peak areas of five distinct peaks in the Raman spectrum of metformin hydrochloride (Figure 2) were recorded on the spectra where the polarization direction of the excitation laser was parallel to the $x y z$ axes (Figure 2). Dataset conversion from $x y z$ to abc component, which were datasets where the polarization direction was parallel to crystallographic abc axes, was performed according to the following equation.

\section{$A=C^{T} \cdot K$}

$A$ and $K$ are the matrices of $x y z$ dataset and $a b c$ component, respectively. $C$ represents the $x y z$ coordinates of unit vectors of $a, b$, and $c$ directions in the unit cell, and $C^{\mathrm{T}}$ is the transposed matrix of $C$.

$$
C^{T}=\left[\begin{array}{ccc}
\frac{\alpha \sin ^{2} \beta}{\sqrt{a^{2} \sin ^{2} \beta+b^{2}}} & \frac{b}{\sqrt{a^{2} \sin ^{2}} \beta+b^{2}} & 0 \\
-\cos \beta & 0 & 1 \\
\frac{b \sin \beta}{\sqrt{a^{2} \sin ^{2} \beta+b^{2}}} & \frac{\alpha \sin \beta}{\sqrt{a^{2} \sin ^{2} \beta}+b^{2}} & 0
\end{array}\right]
$$

$K$ was solved as follows:

$$
K=\left(C^{T}\right)^{-1} \cdot A
$$

Calculations were performed on Microsoft Excel 2013 (Microsoft Corporation, Redmond, WA, USA).

\section{Raman mapping of metformin hydrochloride tablet}

A metformin hydrochloride tablet (METGLUCO ${ }^{\circ} 250 \mathrm{mg}$, Sumitomo Dainippon Pharma, Osaka, Japan) was fixed on a glass plate with instant glue and milled with a milling machine (Proxxon $\mathrm{GmbH}$, Niersbach, Germany) to expose the cross-section. Raman mapping was performed with Renishaw inVia Raman microscope under a $785 \mathrm{~nm}$, $300 \mathrm{~mW}$ excitation laser with $50 \times$ objective lens. The laser exposure time was $1 \mathrm{~s}$ and the mapping area was $250 \mu \mathrm{m} \times 250 \mu \mathrm{m}$ in a spatial resolution of $2 \mu \mathrm{m}$ (15,876 data points in total). The areas of the five distinct peaks of metformin hydrochloride were calculated for each data point and a matrix $\mathrm{B}(15876 \times 5)$ was defined. The abc component of each data point, matrix $\mathrm{D}(15876 \times 3)$, was calculated as follows:

$$
\begin{aligned}
& B=D \cdot K \\
& D=B \cdot K^{T} \cdot\left(K \cdot K^{T}\right)^{-1}
\end{aligned}
$$

\section{Image formation from abc component}

Components in each column of $D$ were then normalized to 0-255 integer to form a matrix $D^{\prime}$. Each column of $D^{\prime}$ was transferred to ImageJ software (National Institutes of Health, Bethesda, MD, USA) as a text image and converted to a picture image. The $a, b$, and $c$ axis component images were colored by red, green, and blue (RGB), respectively, and the three images were merged.

\section{Results and Discussion}

\section{Crystal structure of metformin hydrochloride}

Structure solution and refinement of the metformin hydrochloride crystal were performed as described in the Materials and Methods. A summary of the crystal data is presented in Table 1. It was found that the metformin hydrochloride crystal formed a primitive monoclinic cell and the widest plane was the (1-10) face (Figure 1C). The structural data have been deposited with The Cambridge Crystallographic Data Centre (CCDC: 1433396). Being different from the structure of the phenytoin crystal [10], the unit cell of metformin hydrochloride was not a rectangular solid and was settled with a tilt against morphologically $x y z$ axes (Figure 1D). Thus, it was necessary to perform matrix calculations to resolve the abc component as shown in Materials and Method.

\section{Raman crystal orientation mapping (RCOM)}

In this study, we developed a novel Raman mapping method, RCOM. The abc component on each data point was resolved and converted to $0-255$ integer. The text images corresponding to the $a$, $b$, and $c$ axes were transformed to RGB images and merged. Images obtained in this way (RCOM images) represent how much each component contributes to the Raman spectrum on a data point, and this indicates how much each crystallographic axis is parallel to the polarization direction of the excitation laser (that is, the image reveals the orientation of axes). As described in the Introduction, because the axes' orientation changes on the boundary between primary particles, the RCOM image represents the primary particle image within the aggregated cluster of API. Figure 3B shows the RCOM image of the cross-section of metformin hydrochloride tablet. The difference in color reflects the difference of crystal orientation. For example, the blue region indicates that the $c$-axis is parallel to the polarization direction, and the green region indicates that the $b$-axis is parallel, and the boundary of color is the boundary of primary particles. Thus, we can understand the morphology such as the particle size of API within the aggregation in a solid formulation. Compared with the typical Raman imaging [11] using averaged metformin hydrochloride Raman spectrum as an index (Figure $3 \mathrm{~A}$ ), the RCOM image provided more detailed information of API particle in the solid formulation. 


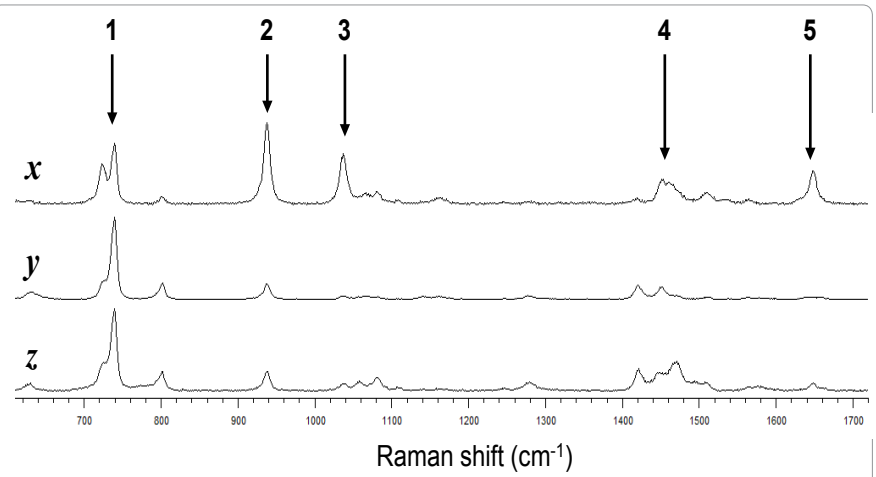

Figure 2: Raman spectra of metformin hydrochloride where the polarization direction of the excitation laser is parallel to $x, y$, and $z$ axes. Peaks 1 to 5 are the target peaks for analysis.

A

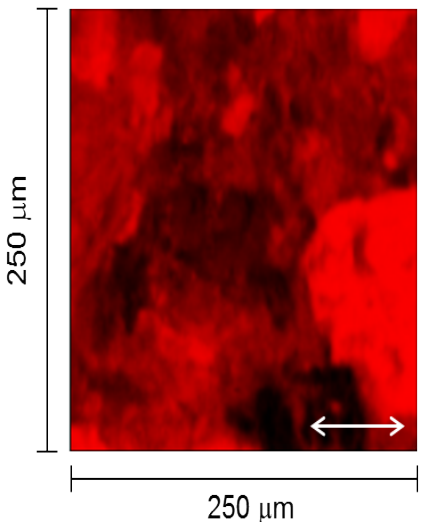

Figure 3: Raman imaging of the cross-section of a metformin hydrochloride tablet $(250 \mu \mathrm{m} \times 250 \mu \mathrm{m}$ area). (A) A typical Raman chemical imaging using averaged metformin hydrochloride Raman spectrum as an index. (B) Imaging by Raman crystal orientation mapping in same area of (A). Double-headed arrow indicates the polarization direction of the excitation laser.

\begin{tabular}{|c|c|}
\hline Molecular formula & $\mathrm{C}_{4} \mathrm{H}_{12} \mathrm{CIN}_{5}$ \\
\hline$a$ & $7.925(8) \AA$ \\
\hline$b$ & $13.907(14) \AA$ \\
\hline$c$ & $7.949(9) \AA$ \\
\hline$a$ & $90.000^{\circ}$ \\
\hline$\beta$ & $114.363(15)^{\circ}$ \\
\hline$V$ & $90.000^{\circ}$ \\
\hline Volume & $798.1(15) \AA^{3}$ \\
\hline Symbol & $P 2_{1} / c$ \\
\hline Number & 14 \\
\hline Centricity & centric \\
\hline$Z$ value & 4 \\
\hline Formula weight & 165.63 \\
\hline Calculated density & 1.378 \\
\hline$\mu$ & $4.153 \mathrm{~cm}^{-1}$ \\
\hline Crystal system & monoclinic \\
\hline laue group & $2 / \mathrm{m}^{\circ}$ \\
\hline lattice type & $P$ \\
\hline
\end{tabular}

Table 1: Unit cell parameters and space group information of metformin hydrochloride crystal.

\section{Application of RCOM imaging to formulation development and quality control}

The RCOM imaging method is applicable to any other API if API is crystalline and the direction of crystallographic axes is clarified using X-ray structural analysis. Whether the solid consists of a pure component or mixture or whether it is before or after tableting is not relevant in recording the ROM image. No special software for calculation is necessary. This imaging method could help formulation development. For example, the distribution of the primary particle size after forming a test tablet could be assessed, which is important information for the dissolving performance of a poorly soluble API. For formulation quality control, it would also provide more useful information, such as the uniformity of the primary particle size or the aggregation states in tablet, than does the typical chemical imaging methods. Assessment of primary particle size distribution of API in tablets based on the RCOM imaging is now under investigation in our laboratory.

\section{Acknowledgement}

The authors are grateful to Renishaw KK (Tokyo, Japan) for technical assistance with Raman spectroscopy. The authors would like to thank Enago (www.enago.jp) for the English language review.

\section{References}

1. Gordon KC, McGoverin CM (2011) Raman mapping of pharmaceuticals. Int J Pharm 417: 151-162.

2. Haaser $M$, Windbergs $M$, McGoverin $C M$, Kleinebudde $P$, Rades $T$, et al (2011) Analysis of matrix dosage forms during dissolution testing using raman microscopy. J Pharm Sci 100: 4452-4459.

3. Hubert S, Briancon S, Hedoux A, Guinet Y, Paccou L, et al. (2011) Process induced transformations during tablet manufacturing: phase transition analysis of caffeine using DSC and low frequency micro-Raman spectroscopy. Int J Pharm 420: 76-83.

4. Henson MJ, Zhang L (2006) Drug characterization in low dosage pharmaceutical tablets using Raman microscopic mapping. Appl Spectrosc 60: 1247-1255.

5. Sasic S, Clark DA (2006) Defining a strategy for chemical imaging of industria pharmaceutical samples on Raman line-mapping and global illumination instruments. Appl Spectrosc 60: 494-502.

6. Sasic S, Clark DA, Mitchell JC, Snowden MJ (2005) Raman line mapping as a fast method for analyzing pharmaceutical bead formulations. Analyst 130 1530-1536.

7. Yamashita M, Sasaki H, Moriyama K (2015) Vapor Phase Alkyne Coating of Pharmaceutical Excipients: Discrimination Enhancement of Raman Chemical Imaging for Tablets. J Pharm Sci.

8. Gamble JF, Hoffmann M, Hughes H, Hutchins P, Tobyn M (2014) Monitoring process induced attrition of drug substance particles within formulated blends. Int J Pharm 470: 77-87.

9. Hoffmann M, Wray PS, Gamble JF, Tobyn M (2015) Investigation into processinduced de-aggregation of cohesive micronised API particles. Int J Pharm 493 341-346.

10. Moriyama K, Furuno N, Yamakawa N (2015) Crystal face identification by Raman microscopy for assessment of crystal habit of a drug. Int J Pharm 480: 101-106.

11. Vajna B, Farkas I, Szabó A, Zsigmond Z, Marosi G (2010) Raman microscopic evaluation of technology dependent structural differences in tablets containing imipramine model drug. J Pharm Biomed Anal 51: 30-38. 